Revista Tecnologia e Ambiente, v. 21, 2014, Criciúma, Santa Catarina. ISSN: 1413-

8131 (versão impressa) ISSN: 2358-9426 (versão eletrônica)

\title{
ANÁLISES DE RISCO E CONFIABILIDADE APLICADAS À GESTÃO DE ESTAÇÕES DE TRATAMENTO DE EFLUENTES INDUSTRIAIS (ETEIS): UMA REVISÃO CRÍTICA
}

\author{
RISK AND RELIABILITY ANALYSIS APPLIED TO THE MANAGEMENT OF \\ INDUSTRIAL WASTEWATER TREATMENT PLANTS (WTP): A CRITICAL REVIEW \\ Shyrlene Lima de Aquino Oliveira ${ }^{1}$ \\ Olavo Barbosa Filho, Gandhi Giordano ${ }^{2}$
}

\section{RESUMO}

As Estações de Tratamento de Efluentes Industriais (ETEIs), desde a etapa de projeto, devem obdecer a critérios de desempenho médio semelhantes àqueles utilizados para projeto de unidades de produção industrial. Deste modo, é fundamental que as indústrias reconheçam as unidades de tratamento de efluentes como plantas industriais e estabeleçam para essas, sistemas de gestão ambiental efetivos, providos de controles que garantam atendimento à legislação ambiental. $O$ presente trabalho tem como objetivo revisar criticamente metodologias que auxiliam na operação estável de ETEIs, centradas nos conceitos de confiabilidade e manutenção industrial. Foram analisadas metodologias de aplicabilidade geral como a técnica FMECA (Failure Modes, Effects and Criticality Analysis), que possibilita hierarquizar os principais riscos do sistema (embora possuindo caráter subjetivo pautado na experiência do técnico analista); e métodos específicos de análise da tratabilidade de efluentes, como o Coeficiente de Confiabilidade (CDC), ferramenta de fácil inserção nas rotinas de monitoramento de ETEIs e que permite quantificar o tempo de atendimento à legislação ambiental (embora não rastreie as possíveis causa da baixa confiabilidade). Após a avaliação individual dos métodos estudados, concluiu-se que as limitações identificadas em cada um desses podem ser sanadas pela aplicação conjunta das metodologias, fornecendo uma sistemática robusta para gestão de efluentes líquidos.

Palavras-chave: Confiabilidade; Efluentes industriais; Manutenção

\begin{abstract}
The Wastewater Treatment Plants (WTP), from the design stage, must comply with criteria average performance similar to those used to project of industrial production units. Therefore, it is critical that industries recognize the wastewater treatment units as industrial plants and establish an effective environmental management system, equipped with controls to ensure compliance with the environmental legislation. This paper aims to critically review methodologies that support the stable operation of WTP, focusing on the concepts of industrial reliability and maintenance. Methods of general applicability were analyzed, such as the FMECA technique (Failure Modes, Effects and Criticality Analysis), which allows to rank the main risks of the system (although being subjective in nature, due to its dependence on the experience of the technical analyst); and methods

\footnotetext{
${ }^{1}$ Mestre em Engenheira Ambiental pela Universidade do Estado do Rio de Janeiro. Engenheira de Meio Ambiente da PETROBRAS (shyrlene_aquino@hotmail.com). Autor para correspondência. Avenida Henrique Valadares, 28 - Centro - Rio de Janeiro - CEP: 20031-030.

2 Professores Associados do Departamento de Engenharia Sanitária e Meio Ambiente - DESMA da Universidade do Estado do Rio de Janeiro - UERJ.
} 
Revista Tecnologia e Ambiente, v. 21, 2014, Criciúma, Santa Catarina. ISSN: 1413-

8131 (versão impressa) ISSN: 2358-9426 (versão eletrônica)

focused specifically on the analysis of wastewater treatability, such as the Coefficient of Reliability, a tool easy to insert in the routine monitoring of WTP, which quantifies the time of compliance with the environmental legislation (although being unable to identify the specific causes of the low reliability). After individual evaluation of the methods studied, it was concluded that the identified limitations can be remedied by application of both methodologies, providing a robust system for managing wastewater. Key-word: Reliability. Maintenance. Wastewater.

\section{INTRODUÇÃO}

O impacto do lançamento de efluentes de Estações de Tratamento de Efluentes Industriais (ETEIs) em corpos d'água sempre foi um motivo de grande preocupação, embora atualmente a legislação brasileira procure influir, tanto nas condições de descarga, quanto no nível de tratamento exigido para minimizar os impactos ambientais negativos provocados pelo lançamento de efluentes líquidos (OLIVEIRA; VON SPERLING, 2005a).

A Resolução CONAMA n 357 , de 17 de março de 2005, que revoga a CONAMA n ${ }^{\circ} 20$, de 1986, estabelece a classificação dos corpos de água e diretrizes ambientais para o enquadramento dos corpos de água superficiais, assim como as condições e padrões de lançamento de efluentes (BRASIL, 2005). No entanto, esta resolução deixou algumas "lacunas" para complementação posterior, conforme pode ser observado no texto do Art. 44: "O CONAMA, no prazo de um ano, complementará, onde couber, condições e padrões de lançamento de efluentes previstos nesta Resolução".

Assim, em 2008, foi publicada a Resolução CONAMA nº 397, que alterava alguns aspectos da CONAMA n $n^{\mathbf{3}}$ 357, tais como a Tabela X e o inciso II do Art. 34 (BRASIL, 2008).

Posteriormente, em 13 de maio de 2011, foi emitida a Resolução CONAMA n ${ }^{\circ}$ 430, que trata das condições e padrões de lançamento de efluentes, assim como complementa e altera a Resolução CONAMA n 357 (BRASIL, 2011).

Um ponto de grande relevância, no que tange à publicação da CONAMA n ${ }^{\circ} 430$, foi o estabelecimento, no Capítulo III, das "Diretrizes para Gestão de Efluentes", que, entre outros aspectos, requerem que as fontes poluidoras dos recursos hídricos devem buscar práticas de gestão de efluentes visando ao uso eficiente da água, redução de geração e melhoria da qualidade dos efluentes (BRASIL, 2011). Adicionalmente, a 
Revista Tecnologia e Ambiente, v. 21, 2014, Criciúma, Santa Catarina. ISSN: 1413-

8131 (versão impressa) ISSN: 2358-9426 (versão eletrônica)

Resolução impõe prazo de 3 anos para que os empreendedores potencialmente poluidores ajustem suas plantas de forma a atender aos requisitos da legislação.

A despeito das imposições legais e das rotas tecnológicas escolhidas para o tratamento dos efluentes, ainda pode ser observada em muitas indústrias a dificuldade prática de atendimento legal.

A observação ressaltada no parágrafo anterior pode ser atribuída a alguns aspectos, tais como: o não reconhecimento do sistema de tratamento de efluentes como uma planta de produção, merecedora da mesma atenção que as unidades geradoras de lucro, o que decorre na inexistência ou ineficácia da gestão dos rejeitos de suas atividades produtivas; as normas e documentos técnicos, geralmente usados como norteadores da estratégia de dimensionamento das ETEIs, que indicam o nível de segurança operacional, mas não garantem a eficiência do tratamento; e as técnicas usualmente indicadas para a definição da eficiência de projetos de estações de efluentes, que não consideram a variabilidade inerente a cada processo e sua associação direta com a confiabilidade (OLIVEIRA, 2014).

As observações destacadas nos parágrafos anteriores remetem à gestão dos efluentes líquidos a importante missão de integrar a operação segura, confiável e eficiente de sistemas de tratamento de efluentes industriais.

Os métodos que permitem avaliar o desempenho de ETEIs e seus elementos críticos se mostram fundamentais para que se alcance os padrões de lançamento exigidos pela sociedade através da regulamentação legal. Isto, por sua vez, requer um desempenho aceitável do tratamento do efluente e uma frequência adequada de manutenção. Tais métodos demonstram, com efeito, um potencial atrativo como ferramentas de gestão ambiental e de riscos.

\section{MÉTODO}

O presente estudo foi baseado na análise bibliográfica das técnicas de Análise de Riscos, Manutenção Centrada na Confiabilidade (MCC), Análise de Modo de Falhas, Efeitos e Criticidade (FMECA), visando à sua aplicabilidade na garantia de confiabilidade de ETEIs.

Com base em teorias previamente estabelecidas para o esclarecimento dos problemas, foi considerado conveniente o uso do método dedutivo. 
Revista Tecnologia e Ambiente, v. 21, 2014, Criciúma, Santa Catarina. ISSN: 1413-

8131 (versão impressa) ISSN: 2358-9426 (versão eletrônica)

O método utilizado contempla um estudo exploratório, por meio do levantamento dos principais estudos teóricos e empíricos que abordam o objeto da pesquisa.

Tendo em vista a escolha de uma pesquisa exploratória, este estudo não teve como objetivo apresentar hipóteses e sim ajudar a estabelecer as prioridades a pesquisar.

\section{GERENCIAMENTO DE RISCOS: VISÃO GERAL}

\subsection{Gestão de Riscos}

$\mathrm{O}$ risco pode ser considerado como a probabilidade de um evento indesejado ocorrer em um período específico ou em determinada circunstância, podendo ser expresso como uma frequência ou uma probabilidade. Em termos matemáticos, frequentemente, é expresso em termos de falha e consequência (SKELTON, 1997).

De Gracia (1998), por sua vez, define risco como sendo o produto da probabilidade de ocorrência de um evento pelas consequências/danos esperados, ou seja:

$$
\mathrm{R}=\mathrm{P} \times \mathrm{D}
$$

Onde:

$\mathrm{R}$ - Risco;

P - Probabilidade de ocorrência de um evento;

D - Valor dos danos associados.

Por fim, a Associação Brasileira de Normas Técnicas (ABNT, 2009) conceitua risco como o efeito da incerteza nos objetivos. O efeito é um desvio em relação ao esperado e os objetivos podem ter aspectos financeiros, ambientais ou relativos à saúde e segurança.

A disponibilidade de ferramentas que minimizem incertezas, ou seja, permitam a identificação e avaliação do que poderá ocorrer durante o andamento de um projeto ou operação de um sistema é fundamental para a obtenção dos resultados desejados. O processo que guia o gestor para as múltiplas tomadas de decisões é o Gerenciamento de Riscos. 
Revista Tecnologia e Ambiente, v. 21, 2014, Criciúma, Santa Catarina. ISSN: 1413-

8131 (versão impressa) ISSN: 2358-9426 (versão eletrônica)

As incertezas não podem ser totalmente eliminadas, mas podem ser reduzidas a um grau considerado tolerável. Em outras palavras, o gerenciamento de risco não garante a não ocorrência de eventos indesejados no decorrer do processo, mas poderá reduzir as incertezas, uma vez que estima a probabilidade de ocorrência de riscos, avalia suas consequências e determina as causas que podem fazer o risco se materializar.

Como resultado, um risco identificado na análise pode ser considerado aceitável ou inaceitável. Cabe aos stakeholders ${ }^{3}$ aceitá-lo ou não, a depender do seu grau de tolerância (PEDROSO, 2007).

A gestão de riscos, portanto, é um processo de identificação e mensuração de riscos, desenvolvimento e escolha das opções de gerenciamento para controle destes riscos (KERZNER, 1998).

Smith e Merritt (2002) afirmam que o gerenciamento ou gestão de riscos está diretamente relacionado ao desenvolvimento de um modelo cujo foco esteja nas principais causas do risco. Este modelo permite a quantificação do impacto do risco, de modo a comparar com outros riscos e hierarquizá-los, contribuindo com os gestores na formulação de planos efetivos para a mitigação ou o bloqueio das causas.

De uma forma geral, a gestão de riscos visa ao controle dos riscos e engloba um conjunto de atividades específicas que tem como intuito a boa governança de uma organização ou processo, sem que eventos indesejáveis e não planejados desvirtuem os seus objetivos e metas (DANTAS, 2010).

Ainda de acordo com Dantas (2010), um dos principais benefícios da gestão de riscos é o desenvolvimento das atividades inerentes a uma organização em um ambiente que controla permanentemente seus riscos, garantindo assim vantagens competitivas e, no caso em análise, atendimento aos aspectos legais e boa imagem da empresa perante os stakeholders. O autor considera adicionalmente que o processo de gerenciamento de riscos tem cinco passos fundamentais:

1. Identificar os Riscos;

2. Analisar os Riscos;

3. Mapear e priorizar os riscos;

\footnotetext{
${ }^{3}$ Stakeholders: o $\mathrm{PMI}^{\circledR}$ no $\mathrm{PMBOOK}^{\circledR}$ define stakeholders como partes interessadas, que podem ser pessoas, organização, clientes e público, desde que estejam envolvidos no projeto.
} 
Revista Tecnologia e Ambiente, v. 21, 2014, Criciúma, Santa Catarina. ISSN: 14138131 (versão impressa) ISSN: 2358-9426 (versão eletrônica)

4. Solucionar os riscos;

5. Monitorar os riscos.

\subsection{Principais Métodos de Análise de Riscos}

Os métodos de análise de riscos visam ao entendimento profundo dos potenciais problemas em projetos ou sistemas. As técnicas podem abranger estimativas quantitativas e medidas para cada risco ou informações qualitativas que classificam os riscos em faixas e categorias (KENDRICK, 2003).

Em muitos casos, os dois métodos podem ser aplicados conjuntamente no processo de identificação e estimação dos riscos do sistema em estudo e, neste caso, a metodologia é denominada de semi-quantitativa.

Os dois métodos, portanto, podem ser complementares e integrados, a partir da identificação de suas especificidades. Os métodos qualitativos fornecem compreensão dos fenômenos e os quantitativos dão valores numéricos das probabilidades e das consequências dos eventos (PARDO, 2009; PEDROSO, 2007).

Técnicas qualitativas apresentam maior facilidade de aplicação e geralmente exigem esforços menores; contudo, não são capazes de fornecer valores numéricos dos riscos e assim não avaliam a importância relativa entre os vários riscos identificados. Por outro lado, as técnicas qualitativas são frequentemente suficientes para a priorização dos riscos e possibilitam gerenciar os riscos que merecem mais atenção naquele momento (KENDRICK, 2003; PEDROSO, 2007).

Métodos quantitativos permitem maior precisão e conhecimento sobre cada um dos riscos; são capazes, inclusive, de avaliar a confiabilidade do sistema. Seguem procedimentos padronizados e objetivos, onde as hipóteses e as variáveis dos problemas estão bem definidas, através de dados quantitativos (KENDRICK, 2003; PARDO, 2009; PEDROSO, 2007).

A escolha do tipo de análise a ser utilizada, qualitativa, semi-quantitativa ou quantitativa, está relacionada à qualidade e quantidade dos dados disponíveis, à natureza do problema e à exatidão desejada. Quando a informação for muito pobre, a utilização de métodos qualitativos ou semi-quantitativos por meio da observação direta dos itens do sistema é aconselhável. Por outro lado, se existir informação que permita uma 
Revista Tecnologia e Ambiente, v. 21, 2014, Criciúma, Santa Catarina. ISSN: 1413-

8131 (versão impressa) ISSN: 2358-9426 (versão eletrônica)

aproximação da realidade, é recomendável fazer análises quantitativas (PARDO, 2009; PEDROSO, 2007).

As principais técnicas de Análise de Riscos estão elencadas na Tabela 1 abaixo:

Tabela 1: Principais métodos e características das análises de risco.

\begin{tabular}{c|c}
\hline MÉTODO DE ANÁLISE DE RISCOS & TIPO DE ANÁLISE \\
\hline Análise por Lista de Verificação (Check List & Qualitativa \\
Anisk $)$ & Qualitativa \\
\hline $\begin{array}{c}\text { Análise Preliminar de Riscos (ARP - Preliminary } \\
\text { Risk }\end{array}$ & Qualitativa \\
\hline $\begin{array}{c}\text { Análise de Perigo e Operacionalidade (HAZOP- } \\
\text { Hazard and Operability Analysis) }\end{array}$ & Semi-quantitativa \\
\hline Análise por Árvore de Eventos (ETA - Event Tree & Semi-quantitativa \\
\hline Análise por Árvore de Falhas (FTA - Fault Tree \\
Analyses)*
\end{tabular}

*Manutenção Centrada na Confiabilidade

\subsection{Confiabilidade e Manutenção Industrial}

A Confiabilidade está associada à operação bem sucedida de um produto ou sistema, sem ocorrência de quebras ou falhas. A análise de confiabilidade permite avaliar a probabilidade do risco ou falha nesses sistemas ou produtos, tornando possível um bom desempenho funcional com baixo índice de falhas (FOGLIATTO; RIBEIRO, 2011; LAFRAIA, 2001).

Em engenharia, é primordial uma definição quantitativa de confiabilidade, principalmente em termos de probabilidade e estatística. Confiabilidade de um item é, portanto, a probabilidade de funcionar de acordo com o especificado no projeto, sem falhas durante o tempo operacional previsto e sob determinadas condições ambientais. 
Revista Tecnologia e Ambiente, v. 21, 2014, Criciúma, Santa Catarina. ISSN: 1413-

8131 (versão impressa) ISSN: 2358-9426 (versão eletrônica)

Sendo assim, a confiabilidade pode ser analisada a partir do objeto de interesse (item), que pode ser um sistema constituído de diversos componentes ou, em casos cujo objetivo é a realização de uma análise detalhada, pode ser um componente do arranjo a ser analisado individualmente (FOGLIATTO; RIBEIRO, 2011).

Outros fatores básicos a serem entendidos nessa definição são ainda os requisitos e propósitos do sistema, o período de tempo previsto para sua vida e as condições ambientais. Com efeito, um mesmo sistema pode ser projetado para tratar efluentes com características diferentes. Como exemplo, um flotador pode ser fabricado para remover sólidos suspensos de efluentes industriais com aspecto oleoso ou ser estruturado para tratamento de efluentes domésticos; o período de tempo deve estar associado à duração da missão, além de considerar que o tempo de uso reduz a sua confiabilidade; e as condições ambientais devem se enquadrar numa faixa razoável (especificada conforme projeto do sistema/produto).

O termo manutenção está relacionado ao setor industrial e seu conceito sofreu diversas alterações à medida que as necessidades das indústrias se tornaram cada vez maiores. Anteriormente, "manutenção" representava o restabelecimento das condições dos equipamentos ou sistemas; contudo, seu conceito dominante hoje é o de procedimento que garante a disponibilidade da função dos equipamentos ou instalações, permitindo a continuidade do processo de produção, com respeito ao meio ambiente, normas de segurança e custos adequados (GURSKI, 2002).

A disponibilidade, segundo Lafraia (2001) é a "medida do grau em que um item estará em estado operável e confiável no início da missão”. A missão é o ato realizado por meio de uma tarefa, com vistas a atingir um objetivo.

Outro conceito que merece atenção é o de mantenabilidade, que difere de manutenção. Aquele é uma característica do projeto que indica a facilidade de manutenção por pessoal de média especialização, sob condições de uso especificadas, considerando o tempo de manutenção e custos. A mantenabilidade é garantida desde que a manutenção seja realizada sob condições determinadas e mediante procedimentos prescritos.

A origem da Manutenção Centrada na Confiabilidade (MCC) está relacionada aos processos tecnológicos e industriais que se desenvolveram após a Segunda Guerra Mundial. Nesse contexto, foram decisivas as pesquisas no setor tecnológico realizadas 
Revista Tecnologia e Ambiente, v. 21, 2014, Criciúma, Santa Catarina. ISSN: 14138131 (versão impressa) ISSN: 2358-9426 (versão eletrônica)

pela indústria bélica americana, seguida pela automação industrial, principalmente para sistemas mecânicos e elétricos, pautada nos avanços da informática e telecomunicações (SIQUEIRA, 2005).

Com o aumento da mecanização da indústria, a partir da década de 50, as máquinas cada vez mais complexas e numerosas necessitavam de maiores cuidados para seu desempenho. Começou então a aflorar a idéia de prevenção de falhas, em detrimento das ações corretivas para reparos em equipamentos, culminando nos anos 60 com a prática de revisões periódicas nas indústrias (RAPOSO, 2004).

Nos últimos 20 anos, a MCC foi disseminada em outras áreas da engenharia e hoje é considerada um método estruturado e sistemático para definição de estratégias de manutenção industrial.

A MCC, segundo Gurski (2002), é o processo capaz de identificar as ações necessárias para assegurar que qualquer item físico continue a desempenhar sua função desejada, dentro do seu contexto operacional. Assim, esta definição possui as seguintes características chave: enfoque sistemático, planejamento de manutenção, confiabilidade e contexto operacional.

Lafraia (2001) salienta que a ênfase deve ser dada na manutenção preventiva, que permite ao sistema o seu funcionamento, e não na tentativa de restauração do equipamento ou sistema a uma condição ideal, até porque é tecnicamente impossível evitar todas as falhas e, mesmo se fosse possível, seria economicamente inviável ou pouco atraente.

O uso dessa ferramenta deve ter início a partir da identificação das funções ou do desempenho requerido pelos itens que compõem o sistema. Difere, portanto, da manutenção tradicional, cujo enfoque é a característica técnica da falha. Posteriormente, há a determinação dos modos de falha e as causas prováveis e, então, o detalhamento dos efeitos e consequências da falha. Com isso, haverá subsídio para avaliar a criticidade das falhas e serem identificadas as consequências relevantes que afetam a segurança, a disponibilidade, o meio ambiente ou custo do sistema em estudo (LAFRAIA, 2001; PARDO, 2009).

As vantagens da aplicação desta técnica estão no decréscimo das rotinas e custos de manutenção preventiva; redução de 30 a $40 \%$ nos custos de mão-de-obra e materiais; garantia de que os recursos da manutenção serão aplicados onde o efeito é maior; 
Revista Tecnologia e Ambiente, v. 21, 2014, Criciúma, Santa Catarina. ISSN: 1413-

8131 (versão impressa) ISSN: 2358-9426 (versão eletrônica)

melhoria das condições ambientais e de segurança; aumento de vida útil do equipamento; compartilhamento dos problemas da manutenção; senso de equipe e motivação de pessoal, entre outros.

Existem vários métodos de MCC, que podem ser baseados em manutenção reativa, preventiva, preditiva ou proativa. Essas estratégias podem ser utilizadas integradas para melhor aproveitamento de seus pontos fortes, de modo a otimizar as instalações e equipamentos e minimizar custos do ciclo de vida (PARDO, 2009). Neste trabalho, será detalhada a técnica de manutenção proativa, denominada Análise por Modo de Falhas, Efeitos e Criticidade (FMECA - Failure mode, Effects and Criticality Analysis), por seu caráter semi-quantitativo.

\subsubsection{Análise por Modo de Falhas, Efeitos (FMEA) e Criticidade (FMECA)}

O método FMECA possibilita uma análise de como os componentes de um equipamento ou sistema podem falhar, assim como a estimativa de taxas de falhas e seus efeitos e o estabelecimento de práticas que permitam aumentar a probabilidade de um equipamento ou sistema funcionar de acordo com o esperado (DE CICCO; FANTAZZINI, 1988).

Representa uma técnica indutiva, estruturada e lógica para diagnóstico (identificação) e prognóstico (antecipação) das causas e efeitos de cada modo de falha de um sistema, produto ou equipamento. Como resultado, devem ser planejadas ações corretivas a serem praticadas de acordo com sua criticidade (LAFRAIA, 2001).

Os autores mencionados nos parágrafos acima e Brito (2007) elencam os principais objetivos do FMECA:

- Antecipação sistemática aos modos de falhas conhecidos ou prováveis, além da recomendação de ações corretivas;

- Revisão documentada de projetos;

- Avaliação lógica de mudanças em projetos, processos ou materiais;

- Determinação dos efeitos que as falhas identificadas terão em outros componentes do sistema;

- Determinação dos componentes, cujas falhas poderiam ter efeito crítico na operação do sistema (Falha de Efeito Crítico); 
Revista Tecnologia e Ambiente, v. 21, 2014, Criciúma, Santa Catarina. ISSN: 1413-

8131 (versão impressa) ISSN: 2358-9426 (versão eletrônica)

- Cálculo de probabilidades de falhas em montagens, sistemas e subsistemas, através do cálculo das probabilidades individuais de seus componentes;

- Redução das probabilidades de falhas em sistemas, a partir do uso de componentes de alta confiabilidade e/ou redundância no projeto;

- Desenvolvimento de uma lista de falhas potenciais, classificadas de acordo com seus efeitos, de modo a priorizar as melhorias do projeto;

- Revisão dos controles atuais;

- Determinação dos itens do sistema que necessitam de controles adicionais.

A FMECA é um aprimoramento da FMEA (Failure Mode and Effect Analysis), na qual a análise crítica é realizada. A criticidade é uma função da severidade, do efeito e da frequência da ocorrência de um evento (MANNAN, 2005).

A análise se baseia na identificação das características críticas do sistema para vários tipos de falhas e permite identificá-las antes que aconteçam, por meio de lista de verificação (check list); seu objetivo é responder três perguntas chave (SLACK et al.,1999):

1. Qual a probabilidade da falha acontecer?

2. Qual seria a consequência da falha?

3. Qual a probabilidade dessa falha ser detectada antes de afetar o meio?

É uma técnica que pode ser utilizada tanto para projetos (em todas as suas fases para identificação das possíveis consequências sobre os componentes ou sobre o sistema, em virtude das falhas hipotéticas do componente) quanto para operação (revisão de sistemas já operacionais, revelando falhas de projeto que não foram identificadas anteriormente). A FMECA não é eficiente para identificar combinações de falhas que resultem em acidentes (PETROBRAS, 199-).

Os resultados, num primeiro momento, são qualitativos; contudo, dados quantitativos podem ser utilizados para expressar uma probabilidade.

Durante a pesquisa bibliográfica, não foi verificada a utilização da FMECA para ETEIs; no entanto, por adotar ações preventivas, baseado em prioridades, aparece como indispensável dentro da estratégia de confiabilidade, principalmente no que tange à prevenção da poluição de recursos hídricos e à política de gestão ambiental.

Os dados laboratoriais, bem como os históricos de manutenção registrados nos relatórios das esquipes de operação são ferramentas imprescindíveis para determinar a 
Revista Tecnologia e Ambiente, v. 21, 2014, Criciúma, Santa Catarina. ISSN: 1413-

8131 (versão impressa) ISSN: 2358-9426 (versão eletrônica)

frequência de ocorrências e têm relação direta com as causas dos modos de falha. Apenas a análise individual desses dados permite elencar várias hipóteses que afetam o desempenho do tratamento.

Como limitação, cabe destacar a subjetividade do método, que pode ser amenizada pelo envolvimento de uma equipe técnica multidisciplinar.

\subsection{Confiabilidade do Tratamento de Efluentes}

A qualidade e variabilidade do efluente de uma ETEI dependem de alguns fatores, tais como: variações na carga afluente; condições ambientais; natureza do efluente a ser tratado; presença de substâncias tóxicas e recalcitrantes; especificidades dos processos de tratamento biológicos; e falhas mecânicas, elétricas e humanas no sistema. Todos estes fatores podem trazer efeitos adversos, como instabilidade nos processos, ocasionando eventuais perdas de especificação do efluente tratado (OLIVEIRA; VON SPERLING, 2005a).

Em termos de desempenho de uma estação de tratamento, a confiabilidade é a porcentagem de tempo em que as concentrações no efluente atendem aos padrões de lançamento, estabelecidos legalmente. Assim, uma ETEI será completamente confiável se não houver violação dos limites preconizados pelas legislações ambientais. A falha do processo de tratamento ocorrerá sempre que o padrão de lançamento for excedido (OLIVEIRA; VON SPERLING, 2005a).

Eisenberg et al. (2001) relacionam a confiabilidade de ETEIs a dois aspectos: a confiabilidade mecânica e a confiabilidade do processo.

A confiabilidade mecânica é determinada a partir da identificação dos componentes mecânicos da planta, cujas falhas podem comprometer a qualidade do efluente final a ser lançado no corpo receptor. Em seguida, é determinada a probabilidade de falhas desses componentes.

Com essa avaliação, é possível quantificar a dependência mecânica de uma ETEI em termos operacionais e os pontos críticos no processo de tratamento, que podem, posteriormente, ser melhorados para aumentar a confiabilidade do processo. A FMECA permite a realização dessas etapas, bem como possibilita a determinação da mantenabilidade e disponibilidade operacional de componentes críticos selecionados de 
Revista Tecnologia e Ambiente, v. 21, 2014, Criciúma, Santa Catarina. ISSN: 14138131 (versão impressa) ISSN: 2358-9426 (versão eletrônica)

ETEIs. Outros métodos de manutenção centrada na confiabilidade também são indicados.

No que tange aos equipamentos, Da-Hin et al. (2008) caracterizam o funcionamento de uma ETEI como um conjunto de instalações elétricas, hidráulicas e mecânicas, capazes de proporcionar a operação de tratamento de efluentes. As falhas verificadas nos equipamentos costumam ser as mais diversas; porém, a grosso modo, podem ser identificadas como vibração excessiva, elevação da temperatura, ruídos anormais, corrosão e sujeira.

Eisenberg et al. (2001) aplicaram a ferramenta "Análise de Componente Crítico" para avaliar a confiabilidade mecânica em instalações piloto de tratamento de efluentes. O objetivo deste tipo de análise é determinar os componentes mecânicos na estação de tratamento que teriam o impacto mais imediato na qualidade do efluente devido à ocorrência de falhas. Para tal, coletaram dados de todos os eventos de manutenção planejada e não planejada, assim como as estatísticas de desempenho (descrevem o tempo de espera entre falhas das unidades de tratamento, o tempo médio entre falha global de componentes e a fração de tempo que uma unidade ou componente estava operando) dos componentes individuais do sistema de tratamento.

Os resultados sinalizaram que a variação na concentração do efluente decorrente das falhas ou da manutenção de componentes mecânicos não tem sido muito significativa (97\% do tempo os componentes das unidades estudadas estavam disponíveis). Em outras palavras, a ETEI teria uma disponibilidade operacional de aproximadamente $100 \%$, observada em um período de longo prazo (01 ano), e sua confiabilidade pode ser definida apenas utilizando-se seus dados de performance.

A confiabilidade do processo ou confiabilidade inerente, segundo Eisenberg et al. (2001), engloba a quantificação da variabilidade do efluente final e a sua determinação pode ser feita com o uso de fundamentos estatísticos associados à análise de frequência. A confiabilidade pode ainda ser caracterizada pela estimativa das distribuições de probabilidades acumuladas, relativas a um contaminante específico, em cada etapa do processo de tratamento. Essas distribuições de probabilidade representam a variabilidade do desempenho do processo e permitem estimar a probabilidade de um processo exceder a uma meta pré-fixada. 
Revista Tecnologia e Ambiente, v. 21, 2014, Criciúma, Santa Catarina. ISSN: 1413-

8131 (versão impressa) ISSN: 2358-9426 (versão eletrônica)

Devido às variações na qualidade do efluente tratado, a estação de tratamento deve ser projetada para produzir uma concentração média efluente abaixo dos padrões de lançamento. Niku et al. (1979) desenvolveram um método que relaciona a concentração média do constituinte (valor de projeto) com os valores limites a serem cumpridos, baseando-se em análises probabilísticas. Esse método permite determinar uma concentração média que garante que a concentração do efluente estará abaixo de certo valor, com um determinado nível de confiabilidade. Este método é conhecido como Coeficiente de Confiabilidade (OLIVEIRA; VON SPERLING, 2005b) e é representado conforme as equações 2 e 3 , a seguir:

$$
\begin{aligned}
& \mathrm{CDC}=\text { Distr. NormalPadão }\left(\mathrm{z}_{1-\propto}\right) \\
& \mathrm{Z}_{1-\alpha}=\left[\frac{-\ln \left(\left(\mathrm{m}_{\mathrm{x}} / \mathrm{x}_{\mathrm{g}}\right)\left(1 / \sqrt{\left(\mathrm{V}_{\mathrm{R}}+1\right)}\right)\right)}{\sqrt{\left(\mathrm{V}_{\mathrm{R}}+1\right)}}\right]
\end{aligned}
$$

Onde:

$\mathrm{m}_{\mathrm{x}}$ : média da concentração do constituinte na saída;

$\mathrm{X}_{\mathrm{s}}$ : concentração padrão requerida para o constituinte;

$\mathrm{V}_{\mathrm{x}}$ : coeficiente de variação $(\mathrm{CV})$ definido como desvio padrão dividido pela média;

$\mathrm{Z}_{1-\alpha}$ : número de desvios-padrão a partir da média de distribuição normal.

O CDC é uma ferramenta acessível, de fácil inserção nas rotinas de monitoramento de estações de tratamento de efluentes e permite quantificar o tempo de atendimento às metas pré-fixadas, sejam estas de atendimento ao projeto da unidade, à legislação ambiental ou aos padrões desenvolvidos pela política da empresa.

Esta última característica apresenta elevada sensibilidade, uma vez que ETEIs que adotam metas equivocadas, diferentes, por exemplo, das especificações do projeto, podem mascarar a confiabilidade, tendo como consequência operação inadequada e baixo desempenho. 
Revista Tecnologia e Ambiente, v. 21, 2014, Criciúma, Santa Catarina. ISSN: 1413-

8131 (versão impressa) ISSN: 2358-9426 (versão eletrônica)

Cabe, contudo, ressaltar que a definição da concentração desejável de determinados poluentes no efluente final em projeto é feita, em geral, considerando valores médios; portanto, é ponto vital considerar a variabilidade ou confiabilidade para definição da meta de eficiência, de forma que ela possa ser alcançada durante a operação.

A grande desvantagem da utilização individual deste método é a não identificação das prováveis causas da baixa confiabilidade, que pode ser compensada com a utilização conjunta da FMECA, permitindo dessa forma análise da confiabilidade e criticidade da ETEI.

\section{CONSIDERAÇÕES FINAIS}

Ambas as ferramentas estudadas apresentam aspectos positivos e negativos, sendo que os últimos inviabilizam sua utilização individual, pois não fornecem a robustez necessária para um sistema de gestão efetivo.

A FMECA possibilita uma análise de como podem falhar os componentes do sistema, estima as falhas, seus efeitos e propõe práticas que permitam aumentar a probabilidade do sistema funcionar de acordo com o esperado. Contudo, para obtenção da criticidade, são utilizados critérios que dependem do julgamento subjetivo do analista.

Já o CDC identifica a confiabilidade do sistema, ou seja, a porcentagem de tempo em que as concentrações esperadas no efluente cumprem com os padrões de lançamento estabelecidos legalmente, sendo, portanto, um importante indicador de desempenho operacional. A desvantagem do método é a impossibilidade de determinar as causas da baixa confiabilidade, ou seja, os componentes críticos do sistema.

O CDC pode ser facilmente complementado pela FMECA, que fornecerá as prováveis causas da baixa confiabilidade do sistema; a FMECA, por sua vez, terá sua eficácia aumentada quando executada por uma equipe multidisciplinar e experiente.

Assim, a contribuição deste estudo reside na geração de informações que podem ser usadas por projetistas e operadores de estações de tratamento de efluentes no diagnóstico e prognóstico de desempenho de ETEIs, considerando o sistema como um todo, trazendo como benefícios menor número de paradas programadas, menor custo de 
Revista Tecnologia e Ambiente, v. 21, 2014, Criciúma, Santa Catarina. ISSN: 1413-

8131 (versão impressa) ISSN: 2358-9426 (versão eletrônica)

manutenção e operação, cumprimento da legislação ambiental, atuação nas causas básicas de paradas não programadas, entre outros.

Cabe, entretanto, o reconhecimento da ETEI como uma unidade de produção, a qual deve possuir um sistema eficaz de manutenção e tratamento de anomalias, uma vez que sua má gestão terá efeitos diretos nos custos empresariais, além de impacto negativo perante os stakeholders.

\section{REFERÊNCIAS}

ABNT. ABNT/ISO/IEC. Guia 73: Gestão de riscos: Vocabulário. 1ª Edição, 2009.

BRITO, J. N. Planejamento e Controle da Manutenção. Apostila do Curso Planejamento e Controle da Manutenção. ABEMEC-MG. Belo Horizonte, 2007.

BOLZANI, H. R. O Efeito da Manutenção e das Condições Operacionais no Desempenho de Estação de Tratamento de Esgoto. 156 f. Dissertação (Mestrado em Engenharia Urbana). Departamento de Engenharia Civil. Universidade Estadual de Maringá. 2011.

BRASIL. Conselho Nacional de Meio Ambiente, CONAMA. Resolução no 20 de 18 de junho de 1986. Estabelece a classificação das águas doces, salobras e salinas do

Território Nacional. Diário Oficial da União, Brasília, DF, p. 11356-11361, jul. 1986.

BRASIL. Conselho Nacional de Meio Ambiente, CONAMA. Resolução no 397 de 3 de

Abril de 2008. Altera o inciso II do $\S 40$ e a Tabela X do $\S 5^{\circ}$, ambos do art. 34 da Resolução do Conselho Nacional do Meio Ambiente- CONAMA no 357, de 2005, que dispõe sobre a classificação dos corpos de água e diretrizes ambientais para o seu enquadramento, bem como estabelece as condições e padrões de lançamento de efluentes. Diário Oficial da União, Brasília, DF, n. 66, p. 68-69, abr. 2008.

BRASIL. Conselho Nacional de Meio Ambiente, CONAMA. Resolução no 357 de 17 de março de 2005. Dispõe sobre a classificação dos corpos de água e diretrizes ambientais para o seu enquadramento, bem como estabelece as condições e padrões de lançamento de efluentes, e dá outras providências. Diário Oficial da União, Brasília, DF, n. 53, p. 58-63, mar. 2005.

BRASIL. Conselho Nacional de Meio Ambiente, CONAMA. Resolução no 430 de 13 de maio de 2011. Dispõe sobre as condições e padrão de lançamento de efluentes complementa e altera a Resolução $\mathrm{n}^{\circ}$ 357, de 17 de março de 2005, do Conselho Nacional do Meio Ambiente-CONAMA. Diário Oficial da União, Brasília, DF, n. 92, p. 89, mai. 2011.

DE CICCO, F.; FANTAZZINI. M.L. Introdução à Engenharia de Segurança de Sistemas. $3^{\mathrm{a}}$ ed. São Paulo: FUNDACENTRO, 1988. 109 p.

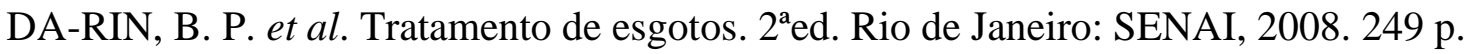


Revista Tecnologia e Ambiente, v. 21, 2014, Criciúma, Santa Catarina. ISSN: 1413-

8131 (versão impressa) ISSN: 2358-9426 (versão eletrônica)

DANTAS, M. L. Avaliação de Riscos em Instalações Portuárias. Olinda: Livro Rápido, 2010. 134 p.

EISENBERG, D. et al. A methodology to evaluate water and wastewater treatment plant reliability. Water Science and Technology. v. 43, n. 10, p. 91-99, 2001.

FOGLIATTO, F. S.; DUARTE, J. L. R. Confiabilidade e Manutenção Industrial. Rio de Janeiro: Elsevier: ABEPRO, 2011.261 p.

KENDRICK, T. Identifying and Managing Project Risk: essential tools for failure Proofing your project. $2^{\text {nd }}$. New York: American Management, 2003.

LAFRAIA, J. R. B. Manual de Confiabilidade, Mantenabilidade e Disponibilidade. Rio de Janeiro: Qualitymark: Petrobras, 2001. 388 p.

MANNAN, S. (Ed.). Lee's Loss prevention in the process industries: hazard identification, Assessment and Control. $3^{\text {rd }}$. v.1. New York: Elsevier, 2005. 307 p.

NIKU, S.; SCHROEDER, E. D.; SAMANIEGO, F. J. Performance of activated sludge processes and reliability - based design. Journal Water Pollution Control Association. v. 51, n. 12, p. 2841-2857. 1979.

OLIVEIRA, S. L. A. Confiabilidade operacional de Estação de Tratamento de Efluentes Industriais (ETEI) de refinaria de petróleo: estudo de caso. 2008. 189 p. Dissertação (Mestrado em Engenharia Ambiental) - Universidade do Estado do Rio de Janeiro, Rio de Janeiro. 2014.

OLIVEIRA, S. M. A. C.; VON SPERLING, M. (2005a) Avaliação de 166 ETEs em operação no país, compreendendo diversas tecnologias. Parte 1: análise de desempenho. Eng. Sanit. Ambiental, Rio de Janeiro. v. 10, n. 4, p. 347-357. 2005a

OLIVEIRA, S. M. A. C.; VON SPERLING, M. Avaliação de 166 ETEs em operação no país, compreendendo diversas tecnologias. Parte 2: influência de fatores de projeto e operação. Eng. Sanit. Ambiental, Rio de Janeiro. v. 10, n. 4, p. 358-368. 2005 b

PARDO, J. A. R. Metodologia para Análise e Gestão de Riscos em Projetos de Pavimentos Rodoviários. 208 f. Dissertação (Mestrado em Geotecnia). Escola de Minas. NUGEO. Universidade Federal de Ouro Preto. Ouro Preto. 2009.

PEDROSO, L. H. Uma Sistemática para a Identificação, Análise Qualitativa e Análise Quantitativa dos Riscos em Projetos. 151 f. Dissertação (Mestrado em Engenharia). Escola Politécnica da Universidade de São Paulo - USP. São Paulo. 2007.

PROJECT MANAGEMENT INSTITUTE. Combined Standards Glossary. $2^{\text {nd }}$ ed. Newton Square. PMI, 2005. 68 p.

PETROBRAS. Técnicas de Análise de Riscos: A.M.F.E. Rio de Janeiro. 199-.

RAPOSO, J. L. O. Manutenção Centrada na Confiabilidade de Sistemas Elétricos: uma proposta para uso de análise de riscos no diagrama de decisão. Dissertação (Mestrado em Engenharia Elétrica). Escola Politécnica. Universidade Federal da Bahia, Salvador. 2005.

SIQUEIRA, I. P. Manutenção Centrada na Confiabilidade: manual de implementação.1 ${ }^{\mathrm{a} e d}$. Rio de Janeiro: Qualitymark, 2005. 408 p. 
Revista Tecnologia e Ambiente, v. 21, 2014, Criciúma, Santa Catarina. ISSN: 14138131 (versão impressa) ISSN: 2358-9426 (versão eletrônica)

SKELTON, B. Process Safety Analysis: an introduction. Gulf Publishing Company, Houston, Texas, 1997. 213 p.

SLACK, N. et al. Administração da Produção. $1^{\text {a }}$ ed. São Paulo: Editora Atlas, 1999. $526 \mathrm{p}$.

SMITH, P. G.; MERRIT, G. M. Proactive Risk Management: controlling uncertainty in product development. productivity press. New York, 2002. 246 p. 